\title{
Distributed Communication-based Model Predictive Control for Long-term Voltage Instability
}

\author{
Mohammad Moradzadeh \\ Electrical Energy Laboratory \\ Dept. of Electrical Energy, \\ Systems and Automation \\ Ghent University \\ 9000 Ghent, Belgium \\ mohammad.moradzadeh@ugent.be
}

\author{
René Boel \\ SYSTeMS Research Group \\ Dept. of Electrical Energy, \\ Systems and Automation \\ Ghent University \\ 9000 Ghent, Belgium
}

\author{
Lieven Vandevelde \\ Electrical Energy Laboratory \\ Dept. of Electrical Energy, \\ Systems and Automation \\ \& Energy knowledge platform Power-Link \\ Ghent University \\ 9000 Ghent, Belgium
}

\begin{abstract}
This paper deals with a form of long-term voltage instability which can arise from uncoordinated control actions taken by interacting voltage controllers. An effective coordination paradigm, based on Model Predictive Control (MPC), is proposed in order to properly coordinate local control actions taken by Load Tap Changing transformers (LTCs). This coordination is achieved by exchange of information on local planned LTC moves among immediate neighboring control agents (CAs) only, within a prediction horizon. Each MPC-based voltage controller, knows only a reduced-order local hybrid system model of its own area, and uses approximate models for its immediate neighboring areas, as well as even more approximate models for remote areas. Simulation results on well-known Nordic32 test system illustrate the good performance of the proposed real-time coordinating voltage controller.
\end{abstract}

\section{INTRODUCTION}

Voltage collapse, as a calamitous result of unmitigated voltage instability, can often lead to blackout in power systems. Over the past 40 years, since the late 1970s, more than 30 major blackouts worldwide were clearly attributed to voltage instability/collapse. Among them, at least 13 voltagerelated blackouts have happened in the USA [1]. In particular, long-term voltage instability was the direct cause of the blackouts in the USA $(08 / 13,14 / 2003)$, Italy $(09 / 28 / 2003)$, Eastern Denmark and Southern Sweden (09/23/2003), Japan (07/23/1987), and Belgium (08/04/1982) [2].

In the context of large-scale multi-area power systems, if the local Control Agents (CAs), (possibly) operated by different Transmission System Operator (TSO), would only use local anticipation some of the loops of interacting dynamical systems may lead to instability of the network as a whole. Indeed it is known that the multi-area electric power system may be destabilized when different neighboring CAs react in an uncoordinated way to incidents that cause the local voltages to temporarily leave their safe sets. An example is the notable incident that Europe's interconnected power grid experienced on November 2006. This incident originated from Northern Germany, where an overhead high voltage transmission line over the river Ems had to be tripped to allow a Norwegian ship to pass safely underneath. This routine tripping led to overloading of the other lines, and eventually the Union for the Coordination of Transmission of Electricity (UCTE) network split into three islands operating with different frequencies for a period of up to two hours. The final report released by UCTE, recently renamed to ENTSO-E (European Network of Transmission System Operators for Electricity), the coordinator of 41 TSOs in 34 European countries, identified three main causes for this incident. Among which was poor interTSO coordination as the other European TSOs did not receive information on the control actions taken by German TSO E.ON Netz [3].

In order to avoid such a global collapse, the local CAs must have some way of anticipating not only how the state variables in its local area will react to the "locally-observed" perturbation and to the planned local control actions, but also how the input port variables generated by neighboring components will evolve in the future.

This paper proposes a solution that combines the advantages of anticipation and coordination. The proposed control scheme assigns to each $\mathrm{CA}$, a local voltage controller based on Model Predictive Control (MPC) for local anticipation, and additionally improves this solution by establishing an active communication architecture on the planned control actions among neighboring CAs in order to avoid the instability in the overall system.

This paper tackles the problem of long-term voltage instability, where interaction (and eventually saturation of) of Load Tap Changing transformers (LTCs), together with the other discrete-event mechanisms such as Over eXcitation Limiter (OXL) of synchronous generators (imposing limits on the maximum reactive power capability), and dynamics of recovery loads are interacting. To cope with this problem, this paper proposes, using a nonlinear hybrid model of the system, a distributed non-cooperative MPC formulation with neighborto-neighbor communication. Each CA knows a local model of its own area as well as reduced-order Quasi Steady-State (QSS) models of its immediate neighboring areas, assuming simpler equivalent PV models for its remote areas. Local decisions are taken using only local measurements and the latest selected control sequences received from the neighboring CAs, by concurrently solving greedy local optimization problems over a finite window in time. The planned local LTC move sequences are then communicated to the immediate neighboring CAs to be taken into account in their next optimization iteration.

The remainder of this paper is organized as follows. The general concept of MPC will be reviewed in section II. The literature review on the existing MPC-based voltage controllers is performed in section III. Section IV describes the proposed 
distributed communication-based MPC (DCMPC) scheme including the modeling framework, formulation of the control problem and the optimization algorithm. Section V presents simulation results obtained from time-domain simulation of the well-known Nordic32 test system, showing the good performance of the DCMPC. Conclusions are drawn in section VI.

\section{Model Predictive Control (MPC)}

One of the most successful classes of closed-loop modelbased schemes is the MPC paradigm, also called receding/moving horizon control. MPC calculates the control action $u\left(t_{k}\right)$ at time $t_{k}=k * T_{c}, k \in \mathbb{Z}^{+}$relying on an estimate of the current system states at discrete time instant $t_{k}$, and on an explicit model of the system. The aim is to predict the future output behavior via simulation over a finite window $\left[t_{k}, \ldots, t_{k+H}\right)$, for a given set of allowable control sequences $\mathbf{u}=\left\{u\left(t_{k}\right), u\left(t_{k+1}\right), \ldots, u\left(t_{k+N-1}\right)\right\}$, where $\mathbf{u} \in \mathcal{U}, 0<$ $N \leq H$, calculating the corresponding performance criterion over horizon $\left[t_{k}, \ldots, t_{k+H}\right)$. At the time instant $t_{k}$, the first element $u^{*}\left(t_{k}\right)$ of the selected best sequence $\mathbf{u}^{*}$ at time $t_{k}$ is then implemented as control input to the system during the interval $\left[t_{k}, \ldots, t_{k+1}\right)$. All these calculations are repeated, using new observations leading to a new state estimate at the next time instant $t_{k+1}$, each time predicting performance over a shifted window with the size of $H * T_{c}$, each time selecting the best control sequence over a new prediction window. At present, MPC is the most-widely used algorithm to deal with multivariable constrained control problems in industry [4][11]. A schematic representation of MPC is shown in Fig. 1. The requirement that a dynamical model must be known is certainly a limitation, especially for electrical power systems, where the loads are not known accurately and where frequent changes of the generation and transmission resources can be expected. However, the inherent feedback of an MPC provides robustness against modeling errors. Moreover, our distributed control design in this paper will only requires, for each local CA, reduced-order dynamic models of its immediate neighboring areas, and not of the remote areas.

As shown in Fig. 2, in order to find the $k^{\text {th }}$ control action $u\left(t_{k}\right)$, MPC operates in successive estimation and optimization steps.

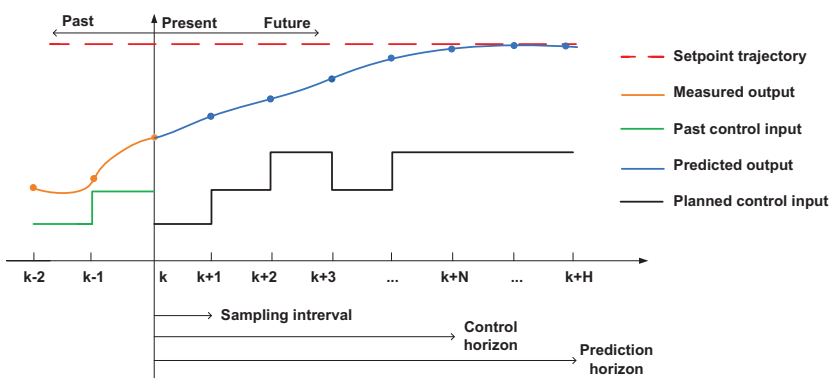

Fig. 1. MPC concept

The prediction horizon $H$ and control horizon $N$ should be selected as the smallest values that lead to a good controller performance. Given sufficiently long prediction horizon $H$, the controller may avoid violating the potential constraints by taking corrective actions immediately.

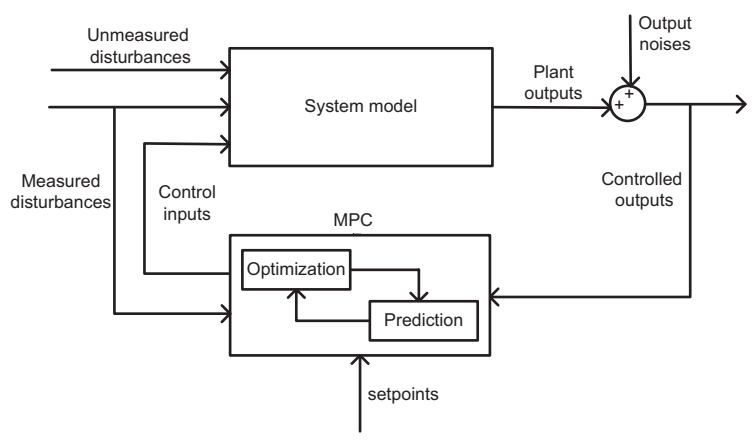

Fig. 2. A system controlled by MPC

It is noteworthy that the concept of "looking ahead/anticipation" is very useful for designing an online coordinating voltage control. The looking-ahead voltage controller can anticipate, within the prediction horizon window $t_{k}, \ldots, t_{k+H}$, for example, the activation of OXLs, moving towards reaching the maximum physical tap limits for LTCs, and deviating too much from the prescribed voltage bounds for buses. The controller will then efficiently use this anticipation, by not selecting a control sequence that causes the violation of said constraints.

\section{MPC-BASED Voltage CONTROL}

The vast majority of the existing MPC-based voltage controllers in the literature are formulated in either a centralized or a completely decentralized fashion. The main drawbacks in the centralized formulation are the huge computational cost, the lack of robustness due to requiring global knowledge of the complete model of the overall system, and the reliability problems due to possible communication failures. Purely decentralized approaches, ignoring interactions among areas, may not lead to a well-performing controller in highly-coupled power systems, leading to suboptimal or even non-convergent performance.

On contrary, this paper proposes the use of distributed wide-area communication-based control approaches, where local optimizations are computionally solved in a distributed manner, while still accounting for the interactions among CAs and preserving the local non-disclosable information e.g. economical cost functions.

In [4], a centralized MPC formulation is performed, using a linearized global system model around an equilibrium, and the underlying optimization problem is solved by a heuristic tree search technique to coordinate generator voltage setpoints, LTC moves and load shedding. Reference [5] solves a centralized MPC optimization, using a single-stage Euler state predictor, and a pseudo-gradient evolutionary programming algorithm to select an optimal combination of the available control inputs. MPC is used in [6] to design a central supervisor which provides setpoints for each local controller, using a pattern search optimization method. A cooperative distributed MPC, using a linear time-invariant model of the system, is applied in [7] to automatic generation control aiming at frequency and tie-line interchange regulation, where each subsystem requires the full knowledge of all other subsystems. A centralized MPC optimization in [8] is solved in a distributed fashion using a classic Lagrangian 
decomposition algorithm to select optimal combinations of generator voltage setpoints and load shedding. Reference [9] employs a centralized MPC formulation, using an explicit model for time evolution of the load power, to select a combination of generator voltage setpoints, shunt capacitors and load shedding to correct non-viable transmission voltages. Load frequency control in interconnected power systems is tackled in [10] by using a decentralized MPC formulation. A so called "almost" decentralized Lyapunov-based MPC algorithm is used in [11] for asymptotic stabilization of the network frequency.

\section{Distributed Communication-BAsed Model Predictive Control (DCMPC)}

\section{A. Modeling Framework}

We define the overall multi-area power system as a graph $\mathcal{G}=(\mathcal{V}, \mathcal{E})$ of $M$ interacting areas $A_{i}$, $i \in \mathcal{A}=\{1, \ldots, M\}$, where each corresponding control agent $\mathrm{CA}_{i}, i \in \mathcal{I}=\{1, \ldots, M\}$, is assigned to a vertex $\nu_{i} \in \mathcal{V}=\left\{\nu_{1}, \ldots, \nu_{M}\right\}$, and the interconnection (and thus communication links) among CAs are represented by a set of edges $\mathcal{E} \subseteq\left\{\left(\nu_{i}, \nu_{j}\right) \in \mathcal{V} \times \mathcal{V} \mid i \neq j\right\}$.

For each $\mathrm{CA}_{i}, i \in \mathcal{I}$, associated to vertex $\nu_{i} \in \mathcal{V}$, consider the sets of indices $\mathcal{N}_{i}=\left\{j \mid\left(\nu_{i}, \nu_{j}\right) \in \mathcal{E}, j \neq i\right\}$ corresponding to the immediate neighbors (directly interconnected through tie-lines), and $\mathcal{R}_{i}=\left\{r \mid\left(\nu_{i}, \nu_{r}\right) \notin \mathcal{E}, r \neq i \neq j\right\}$ to the remote neighbors (indirectly interconnected), where $i \cup \mathcal{N}_{i} \cup \mathcal{R}_{i}=\mathcal{A}=\mathcal{I}$.

This decomposition of the overall system, in this paper, is based on the geographical structure of the system, where a set of buses located at a relatively short electrical distance from each other are considered as one particular area.

Each area $A_{i}, i \in \mathcal{A}=\{1, \ldots, M\}$ is then modeled as a hybrid dynamical system, represented by an input-output hybrid automaton [12]. The HA representation of an LTC in a distributed control fashion $S_{\text {LTC }}$, and of an integral-type OXL $S_{\text {OXL }}$, can be found in [13].

The QSS approximation of the hybrid behavior of each area $A_{i}, i \in \mathcal{A}=\{1, \ldots, M\}$ is expressed by a mixed discreteevent continuous differential-algebraic equations, subject to some local constraints. These nonlinear system equations are often discretized to obtain the following discrete-time control model of each area:

$$
\begin{aligned}
& x_{i}(k+1)=f_{i}\left(x_{i}(k), z_{i}(k), y_{i}(k)\right), k \in \mathbb{Z}^{+} \\
& z_{i}\left(T_{e}^{+}\right)=Z_{i}\left(x_{i}\left(T_{e}^{-}\right), z_{i}\left(T_{e}^{-}\right), y_{i}\left(T_{e}^{-}\right), u_{i}\left(T_{e}^{-}\right)\right) \\
& z_{i}(k)=z_{i}\left(T_{e}^{+}\right), T_{e} \leq k<T_{e+1} \\
& g_{i}\left(x_{i}(k), z_{i}(k), y_{i}(k), \phi_{i}\left(y_{\mathcal{N}_{i}}(k), u_{\mathcal{N}_{i}}^{*}(k-1)\right)=0\right. \\
& h_{i}\left(x_{i}(k), z_{i}(k), y_{i}(k)\right) \leq 0
\end{aligned}
$$

where, $x_{i}$ denotes the local dynamic continuous states of the generators, Automatic Voltage Regulators (AVRs), OXLs and load dynamics in area $A_{i}, i \in \mathcal{A}=\{1, \ldots, M\}, z_{i}$ the discrete-event state variables typically arising from discrete control logic such as thresholds reached by OXLs, LTC tap positions, switched $\mathrm{CBs}$ and disturbances, $T_{e}$ the time at which a discrete event $e$ occurs, $T_{e}^{-}=\lim _{\epsilon \rightarrow 0} T_{e}^{-}-\epsilon$ the pre-event time, $T_{e}^{+}=\lim _{\epsilon \rightarrow 0} T_{e}^{+}+\epsilon$ the post-event time, $y_{i}$ the local algebraic state variables e.g. network voltages and currents, $u_{i}$ the discrete local control inputs. LTC tap position changes is the considered control in this paper. The equality constraint $g_{i}($.$) in (1c) corresponds to the algebraic power$ flow equations. The inequality constraint $h_{i}($.$) in (1d) includes$ physical unviolatable limits (hard constraints) e.g. limits on the tap positions of the LTCs, and/or penalizable operational limits (soft constraints) e.g. predefined voltage bounds.

Note that $\phi_{i}(.,$.$) has been appended into g_{i}$ in (1c) to represent the interaction with the directly connected neighboring areas $A_{j}, j \in \mathcal{N}_{i}$. This means that the effect of the dynamic states and control actions taken by the immediate neighbors on the state evolution of area $A_{i}, i \in\{1, \ldots, M\}$ is implicitly reflected through the instantaneously changing algebraic variables (among which are the boundary bus voltages). Moreover the remote neighbors $A_{r}, r \in \mathcal{R}_{i}$ (the neighbors of neighbors) do not even explicitly appear in (1c), instead their possible effect is incorporated in the nonlinear function $\phi_{i}(.,$.$) of the$ immediate neighboring areas $A_{j}, j \in \mathcal{N}_{i}$.

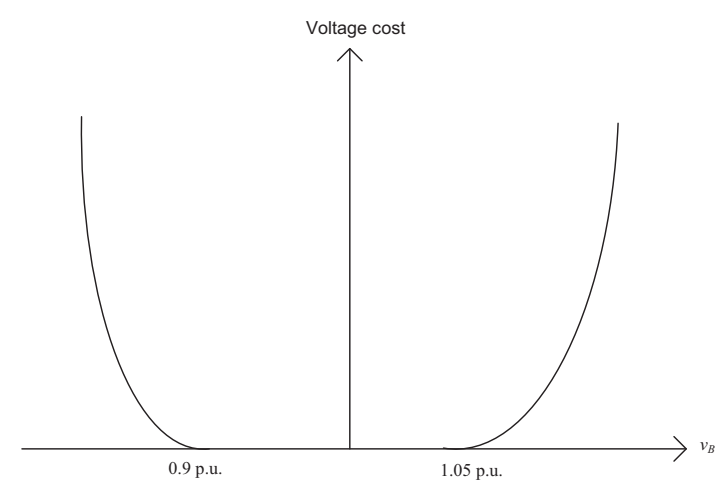

Fig. 3. A simple quadratic cost function

\section{B. Control Problem Formulation}

Each $\mathrm{CA}_{i}, i \in\{1, \ldots, M\}$ is controlled in this paper by a local controller $\mathrm{MPC}_{i}, i \in\{1, \ldots, M\}$. The sequence of predicted state and control values at time $k+\ell, \ell \in\{1, \ldots, H\}$ for $\mathrm{MPC}_{i}, i \in\{1, \ldots, M\}$, based on the information available at time instant $k$ is denoted, respectively, by $\mathbf{x}_{\mathbf{i}}$ and $\mathbf{u}_{\mathbf{i}}$, where

$$
\begin{aligned}
& \mathbf{x}_{i}(k)=\left\{x_{i}(k+1 \mid k), \ldots, x_{i}(k+H \mid k)\right\} \\
& \mathbf{u}_{i}(k)=\{\underbrace{u_{i}(k \mid k), \ldots, u_{i}(k+N-1 \mid k)}_{\mathrm{N}}, \underbrace{0, \ldots, 0}_{\mathrm{H}-\mathrm{N}+1}\}
\end{aligned}
$$

For each $\mathrm{MPC}_{i}, i \in\{1, \ldots, M\}$ with the identical $H$ and $N$, let $\mathbf{u}_{i} \in \mathcal{U}_{i}$ denote a candidate control sequence of LTC tap position changes, where $\mathcal{U}_{i}=\{0,+1,-1\}^{N} \times\{0\}^{H-N+1}$ is the corresponding finite set of all admissible control sequences, with $0,+1$ and -1 referring resp. to having no tap movement, 
an upward tap movement, and a downward tap movement. Note that no tap movement is considered in the interval $\left[t_{k+N}, \ldots, t_{k+H}\right)$.

The main objective of $\mathrm{CA}_{i}, i \in\{1, \ldots, M\}$ is to maintain at time $t$ its $B_{i}$ voltage magnitudes $v_{B}^{i}(t)$ at buses $B \in$ $\left\{1, \ldots, B_{i}\right\}$ within prescribed bounds $v_{\min }^{i} \leq v_{B}^{i}(t) \leq v_{\max }^{i}$ close to the nominal bus voltages. In this paper, we consider $v_{\min }^{i}=0.9 \mathrm{pu}$ and $v_{\max }^{i}=1.05 \mathrm{pu}$ for all CAs. A simple quadratic cost, as shown in Fig. 3, is employed to penalize the voltage deviations for the buses. The secondary objective is to minimize the changes of tap positions $n_{L}^{i}$ in its $L_{i}$ LTCs $L \in\left\{1, \ldots, L_{i}\right\}$ as they cause transients on the system voltages as well as mechanical wear on the LTCs themselves. The distributed non-cooperative MPC-based control algorithm with neighbor-to-neighbor communication for each $\mathrm{MPC}_{i}$, $i \in\{1, \ldots, M\}$ at time instant $k$ can be then formulated as the following optimization problem:

$$
\min _{\mathbf{u}_{i}(k) \in \mathcal{U}_{i}} J_{i}\left(\mathbf{x}_{i}(k), \mathbf{u}_{i}(k) ; x_{i}(k)\right)
$$

where

$$
J_{i}=\sum_{t_{k}}^{t_{k+H}} \Delta u_{i}^{T} \Gamma_{i} \Delta u_{i}+\int_{t_{k}}^{t_{k+H}}\left(\rho_{i}^{T} \Lambda_{i} \rho_{i}\right) d t
$$

subject to for all $k \leq \ell \leq k+H-1$

$$
\begin{aligned}
& x_{i}(\ell+1 \mid k)=f_{i}\left(x_{i}(\ell \mid k), z_{i}(\ell \mid k), y_{i}(\ell \mid k)\right) \\
& z_{i}\left(T_{e}^{+}\right)=Z\left(x_{i}\left(T_{e}^{-}\right), z_{i}\left(T_{e}^{-}\right), y_{i}\left(T_{e}^{-}\right), u_{i}\left(T_{e}^{-}\right)\right) \\
& z_{i}(\ell)=z_{i}\left(T_{e}^{+}\right), T_{e} \leq \ell<T_{e+1} \\
& g_{i}\left(x_{i}(\ell), z_{i}(\ell), y_{i}(\ell), \phi_{i}\left(y_{\mathcal{N}_{i}}(\ell), u_{\mathcal{N}_{i}}^{*}(\ell-1)\right)=0\right. \\
& n_{\text {min }}^{i} \leq n_{L}^{i}\left(z_{i}(\ell)\right) \leq n_{\text {max }}^{i}, L \in\left\{1, \ldots, L_{i}\right\}
\end{aligned}
$$

$\Gamma_{i}=\operatorname{diag}\left(\gamma_{1}, \ldots, \gamma_{L_{i}}\right)$ and $\Lambda_{i}=\operatorname{diag}\left(\lambda_{1}, \ldots, \lambda_{B_{i}}\right)$ are the non-negative diagonal weighting matrices for $\mathrm{MPC}_{i}, i \in \mathcal{I}$, to penalize the amount of tap position changes $\Delta u_{i}$ in its $L_{i}$ LTCs, and the voltage magnitude deviations in its $B_{i}$ buses, respectively. Note that the soft constraints on the $B_{i}$ bus voltage magnitudes $v_{B}^{i}$ are mathematically relaxed by introducing the slack variable penalizing the potential violation of the voltage constraint due to e.g. an unavoidable large disturbance, while the hard constraint on the physical tap positions $n_{L}^{i}$ can never be violated.

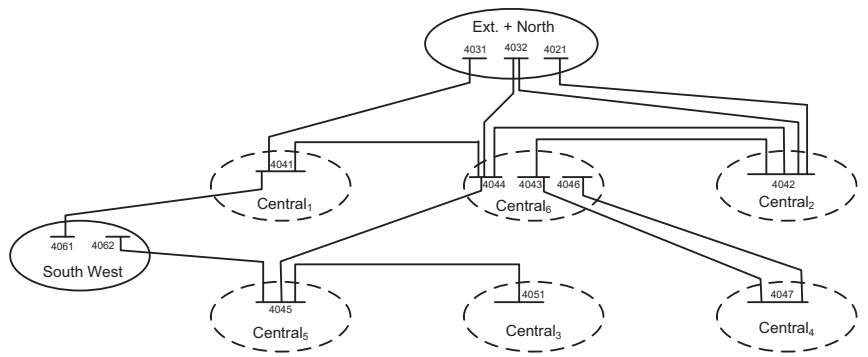

Fig. 4. Nordic32 partitioned interconnected test system

We employ the same reduced-order QSS models [14] for the local $\mathrm{CA}_{i}, i \in \mathcal{I}$ as well as for the immediate neighboring
$\mathrm{CA}_{j}, j \in \mathcal{N}_{i}$. However, a simple PV approximation [15] is used to represent the distant/remote $\mathrm{CA}_{r}, r \in \mathcal{R}_{i}$ as buses with constant voltage magnitudes and constant active power consumptions over the prediction horizon $H$. Note that the reduced-order models for the immediate neighboring areas and for the distant areas are assumed to be given in the simulations as we do not deal in this paper with their identification/estimation.
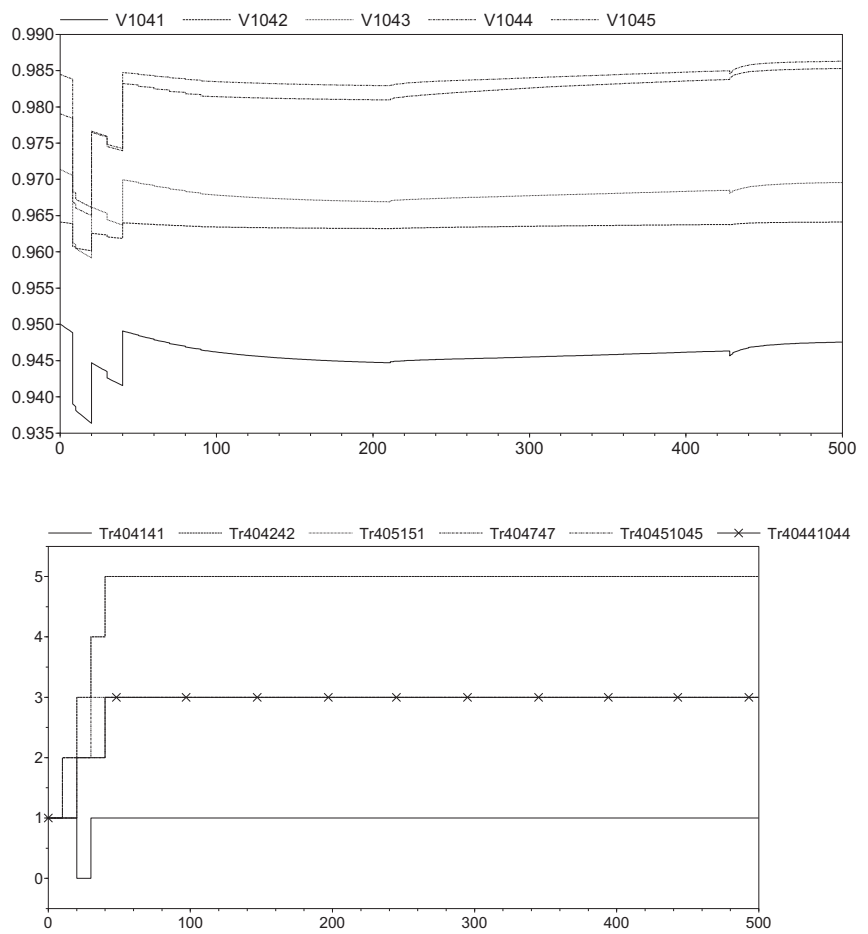

Fig. 5. Transmission bus voltages, and coordinated LTC moves

\section{Optimization algorithm}

The following algorithm is employed by $\mathrm{CA}_{i}$ to solve the DCMPC optimization problem for $\mathrm{MPC}_{i}, i \in\{1, \ldots, M\}$ :

1) $k=0$

2) Initialize with $u_{i}^{*}(k-1), \mathbf{u}_{\mathcal{N}_{i}}^{*}(k-1), \mathbf{u}_{\mathcal{R}_{i}}^{*}(k-$ $1), x_{i}(k), \tilde{x}_{\mathcal{N}_{i}}(k)$

3) Enumerate the discrete set of possible sequences $\mathcal{U}_{i}$ and compute the corresponding costs $J_{i}\left(\mathbf{x}_{i}(k), \mathbf{u}_{i}(k) ; x_{i}(k)\right)$

4) Select the best sequence $\mathbf{u}_{i}^{*}(k)$ and obtain its first element $u_{i}^{*}(k)$

5) Apply $u_{i}^{*}(k)$ to $\mathrm{CA}_{i}$ until next time instant $k+1$

6) Obtain the state estimate $x_{i}(k+1)$ at time $k+1$

7) Communicate $\mathbf{u}_{i}^{*}(k)$ to the immediate neighboring $\mathrm{MPC}_{j}, j \in \mathcal{N}_{i}$

8) $\quad k:=k+1$; go back to step 3 .

Note that the DCMPC approach requires no information exchange on the local state trajectories. However $\tilde{x}_{\mathcal{N}_{i}}(k)$ is the state estimate at time $t_{k}$ of the neighbors that $\mathrm{MPC}_{i}$ uses, based on the QSS reduced-order models of its neighbors, in 

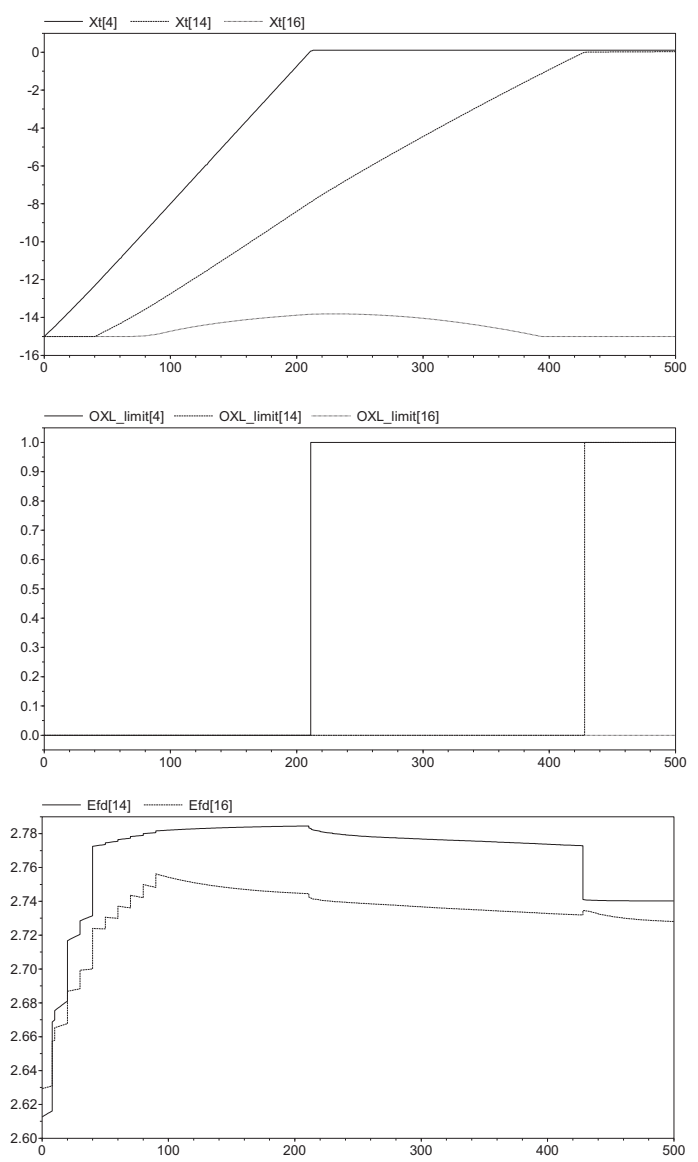

Fig. 6. Inverse-characteristic timers, OXL signals, and generators field voltage

order to compute its own solutions.

\section{Simulation Results}

This section demonstrates the performance of the DCMPC scheme on the Nordic32 test system, following outage of line $4032-4044$ at $t=10 \mathrm{~s}$. The detailed one-line diagram of the Nordic32 system can be found in [13], and only its decomposed areas is shown in Fig. 4. The response of the Nordic32 to this disturbance under DCMPC scheme will be compared with the scenario where LTCs are controlled under local decentralized deadband approach.

The long-term time evolution of the transmission voltage magnitudes at the four most affected buses 1041, 1042, 1043 and 1044 as well as the coordinated LTC moves are shown in Fig. 5. The voltage decline is due to the effect of LTCs trying to restore the distribution side voltages of the LTC-controlled buses as well as OXLs activation of field-current-limited generators restricting their reactive power generation. This proposed set of controls successfully maintains the voltages within the limits, leading to the activation of OXLs over only two generators: $g 4$ at $t=211.1 \mathrm{~s}$ and $g 14$ at $t=427.8 \mathrm{~s}$, as shown in Fig. 6. This occurs when the corresponding timer signal $x_{t}$ become positive. Note that $i_{\mathrm{fd}}$ in generator $g 16$ is kept well below its limit $E_{\mathrm{fd}(\mathrm{lim})}=2.74 \mathrm{pu}$ and thus $g 16$ does not become limited.
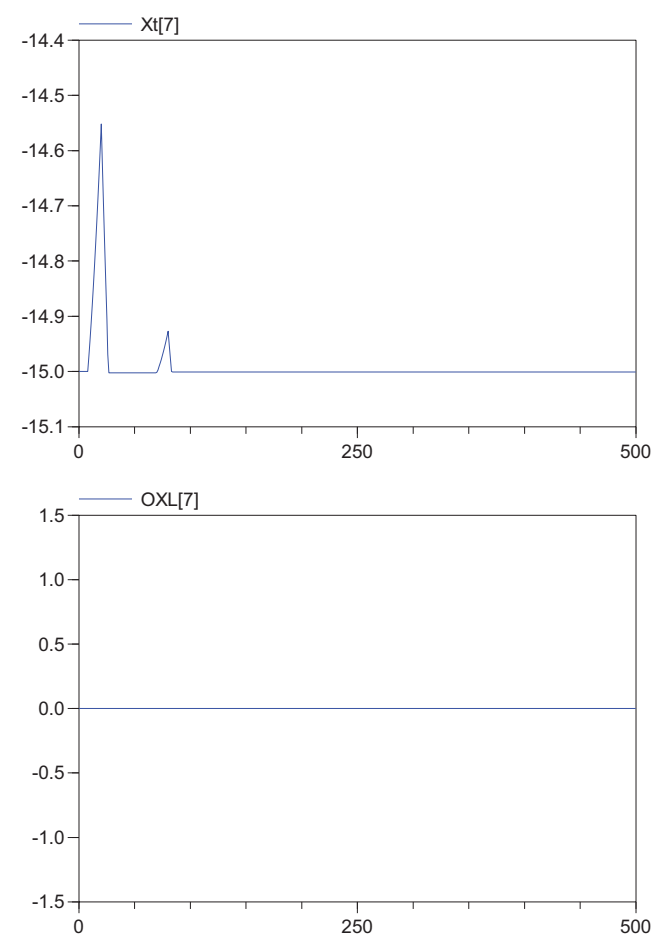

Fig. 7. Inverse-characteristic timer $x_{t}$ and OXL signal of $g 7$

This is thanks to the anticipation of the activation of OXLs, and taking coordinated local control actions with the neighbors, that do not force LTCs to move towards reaching their maximum physical tap limits. As an example the inverse-characteristic timer signal $x_{t}$ of OXL over $g 7$ within Central $_{5}$ is shown in Fig. 7, which initiates twice at $t=8$ and $67 \mathrm{~s}$. This is initially "seen" by the local MPC for Central $_{5}$ in advance, and in an effort to correct this, the local LTC $\operatorname{Tr} 40451045$, takes an upward tap move at $t=20$. Furthermore, at $t=80 \mathrm{~s}$, the local LTC coordinates its upward move with its immediate neighbors, and as a result $g 7$ does never become limited.

This is a significant improvement over the uncoordinated deadband operation of LTCs that leads to a final collapse, as shown in Fig. 8. Here LTCs use only local voltage measurements, and act on the basis of a local deadband $d b=0.02$ p.u., and tap positions are changed accordingly after a time delay $T_{\text {delay }}=10 \mathrm{~s}$. This uncoordinated set of LTC moves trigger the activation of OXLs over six generators $g 13, g 14, g 15, g 16, g 4$ and $g 17$ at $t=100.8,105.1,145.2,160.8,175.8$ and $226.7 \mathrm{~s}$, respectively, and final collapse occurs soon after $g 17$ becomes limited. The LTCs $\operatorname{Tr} 405151$ in $\mathrm{Central}_{3}, \operatorname{Tr} 404242$ in Central 2 , $\operatorname{Tr} 404747$ in Central $_{4}$ and $\operatorname{Tr} 404141$ in Central $_{1}$ reach their maximum limits at times around $t=110,120,120$ and $130 \mathrm{~s}$.

\section{CONCLUSION}

This paper has developed a design methodology for voltage control based on distributed MPC as a tool for coordinating LTCs in adjacent control areas. More specifically, using a nonlinear hybrid model of the system, a distributed non- 

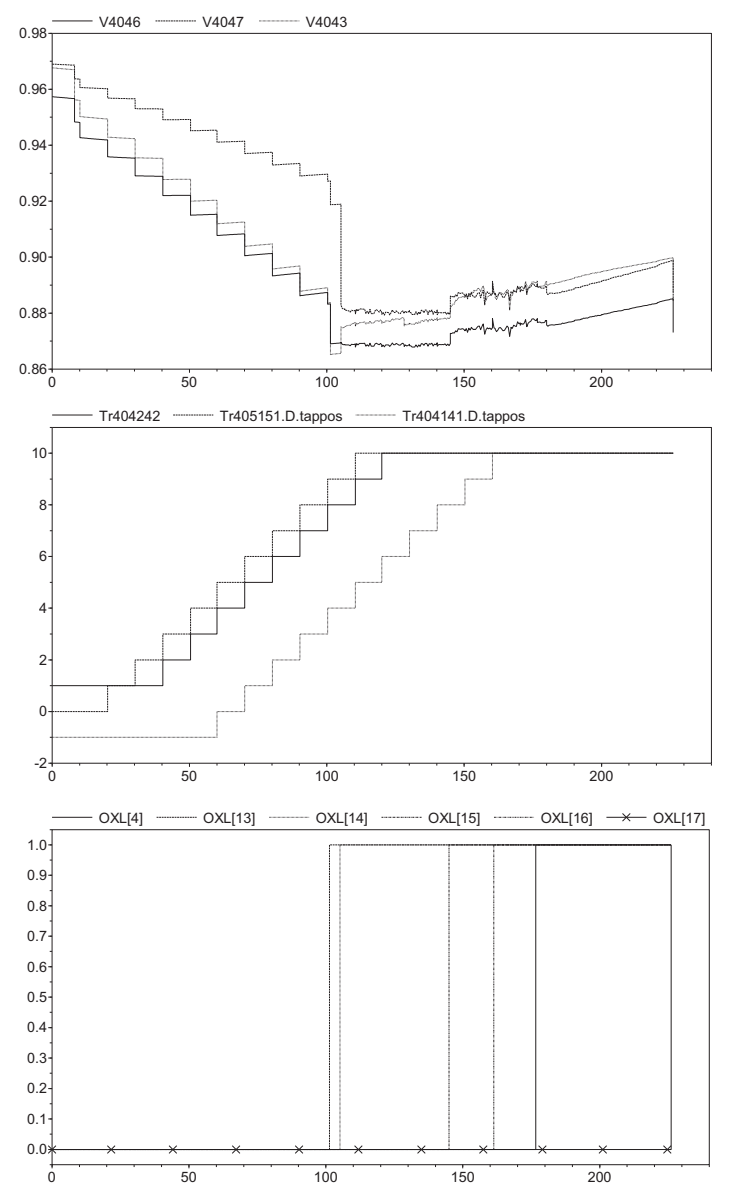

Fig. 8. Bus voltages and uncoordinated deadband LTC moves

cooperative MPC formulation with neighbor-to-neighbor communication is proposed for long-term voltage control of largescale multi-area power systems. Each CA knows a local model of its own area as well as reduced-order models of its immediate neighboring CAs, assuming simpler equivalent models for its remote neighbors. Local decisions are taken by concurrently solving finite-horizon greedy local optimization problems, using only local measurements and the latest selected control sequences received from the immediate neighboring CA. The planned local control sequences are then communicated to the immediate neighboring CAs to be taken into account in their next optimization iteration. The limited amount of exchanged information makes the approach more resilient to communication failures. Furthermore, the fact of not requiring knowledge of the overall system, provides enough robustness against lack of some system information. Simulations on the Nordic32 test system show that the proposed control strategy can stabilize the system in cases when a completely decentralized deadband strategy, without any communications, leads to collapse.

The CPU time required to complete a simulation experiment consists of two distinct terms; the time that the local MPCs take to calculate their optimal control actions at each discrete time instant, plus the time needed to simulate the physical system after applying those calculated control actions in order to obtain the state variables at the end of that control interval. This overall CPU time turned out to be about $3 \mathrm{~s}$ for Nordic32 test system. Taking into account that each CA takes decisions at every $10 \mathrm{~s}$, this ensures that the approach well meets the requirement for online voltage control.

The DCMPC scheme may also be applied as a tool for designing controllers for medium voltage microgrids including distributed generations (DGs) and storage devices. Analogous to the proposed concept, a multi-area small-scale power system can be determined, within the microgrid, by considering, for example, each feeder as an area, and assigning a local MPC to it. The extended economic dispatch problem of microgrids(including the optimal timing for on/off switching of controllable loads, scheduling (postponing/advancing the charge/discharge timing of storage devices even including batteries in EVs, or adjusting the power to heat ratio of CHCP units) can be effectively then tackled by the DCMPC coordination paradigm. In this case, the communication may include the variable import/export electricity pricing among cells, over a prediction horizon in future.

\section{REFERENCES}

[1] Y. Mansour, "Voltage stability," in Power System Dynamics and Stability, R. G. Farmer, Ed. CRC Press LLC, 2001.

[2] G. Andersson, P. Donalek, R. Farmer and et al., "Causes of the 2003 major grid blackouts in North America and Europe, and recommended means to improve system dynamic performance," IEEE Trans. on Power Syst., vol. 20, no. 4, pp. 19221928, Nov. 2005.

[3] Union for the Co-ordination of Transmission of Electricity (UCTE), Final report on system disturbance on 4 november 2006, Jan. 2007.

[4] M. Larsson and D. Karlsson, "Coordinated system protection scheme against voltage collapse using heuristic search and predictive control," IEEE Trans. Power Syst., vol. 18, no. 3, pp. 1001-1006, Aug. 2003.

[5] J.Y. Wen, Q.H. Wu, D.R. Turner and et al., "Optimal coordinated voltage control for power system voltage stability," IEEE Trans. Power Syst., vol.19, no.2, pp. 1115- 1122, May 2004.

[6] R.R. Negenborn, S. Leirens, B. De Schutter and et al., "Supervisory nonlinear MPC for emergency voltage control using pattern search," Control Engineering Practice, vol. 17, no. 7, pp. 841-848, July 2009.

[7] A. N. Venkat, I. A. Hiskens, J. B. Rawlings and et al., "Distributed MPC strategies with application to power system automatic generation control," IEEE transactions on control systems technology, vol.16, no.6, pp.1192-1206, Nov. 2008.

[8] A. Beccuti, T. Demiray, G. Andersson and et al., "A Lagrangian decomposition algorithm for optimal emergency voltage control," IEEE Trans. Power Syst., vol. 25, no. 4, pp. 17691779, Nov. 2010.

[9] M. Glavic, M. Hajian, W. Rosehart and et al., "Receding-Horizon Multi-Step Optimization to Correct Nonviable or Unstable Transmission Voltages," IEEE Trans. Power Syst., vol.26, no.3, pp.1641-1650, Aug. 2011.

[10] T. H. Mohamed, H. Bevrani, A. A. Hassan and et al., "Decentralized model predictive based load frequency control in an interconnected power system," Energy Conversion and Management, vol. 52, issue 2, pp. 12081214, Feb. 2011.

[11] R. M. Hermans, M. Lazar, A. Jokić and et al., "Almost decentralized model predictive control of power networks," In Proc. MELECON2010, pp. 1551-1556, April 2010.

[12] M. Moradzadeh and R. Boel, "A Hybrid Framework for Coordinated Voltage Control of Power Systems," In Proc. IPEC2010, pp. 304-309, Singapore, 2010.

[13] M. Moradzadeh, R. Boel and L. Vandevelde, "Voltage Coordination in Multi-Area Power Systems via Distributed Model Predictive Control," IEEE Trans. Power Syst., vol. 28, no. 1, pp. 513-521, Feb. 2013.

[14] M. Moradzadeh, L. Bhojwani and R. Boel, "Coordinated Voltage Control via Distributed Model Predictive Control," In Proc. CCDC2011, pp. 1612-1618, China,2011.

[15] Y. Phulpin, M. Begovic, M. Petit and et al., "Evaluation of Network Equivalents for Voltage Optimization in Multi-Area Power Systems," IEEE Trans. on Power Syst., vol. 24, no. 2, pp. 729-743, May 2009. 